\title{
Oxybutynin vermindert übermäßiges Schwitzen
}

\begin{abstract}
Für Menschen, die übermäßig schwitzen, scheint laut einer aktuellen randomisierten placebokontrollierten Studie niedrigdosiertes Oxybutynin eine effektive Therapieoption zu sein.
\end{abstract}

Dass das Anticholinergikum Oxybutynin auch an den Schweißdrüsen wirkt und eine übermäßige Schweißproduktion drosseln kann, ist seit Längerem bekannt. Untersuchungen dazu gab es bislang nur wenige. Nun reichen französische Dermatologen eine zwar kleine, aber randomisierte placebokontrollierte Studie nach.

Die Dermatologen teilten zwischen Juni 2013 und Januar 2014 insgesamt 62 Patienten mit primärer überwiegend generalisierter (83\% der Probanden) Hyperhidrose nach dem Zufallsprinzip der Verum- und Placebogruppen zu. Die Oxybutyningruppe startete mit einer Tagesdosis von $2,5 \mathrm{mg}$, die schrittweise auf 7,5 mg pro Tag gesteigert wurde. Die Tagesdosis lag damit deutlich unter der Dosierung von 10-15 mg, die bei der Behandlung der überaktiven Blase üblich ist. Als primären Endpunkt hatten die Dermatologen eine Verbesserung auf der HyperhidroseSchweregrad (HDSS)-Skala um mindestens einen Punkt 6 Wo-

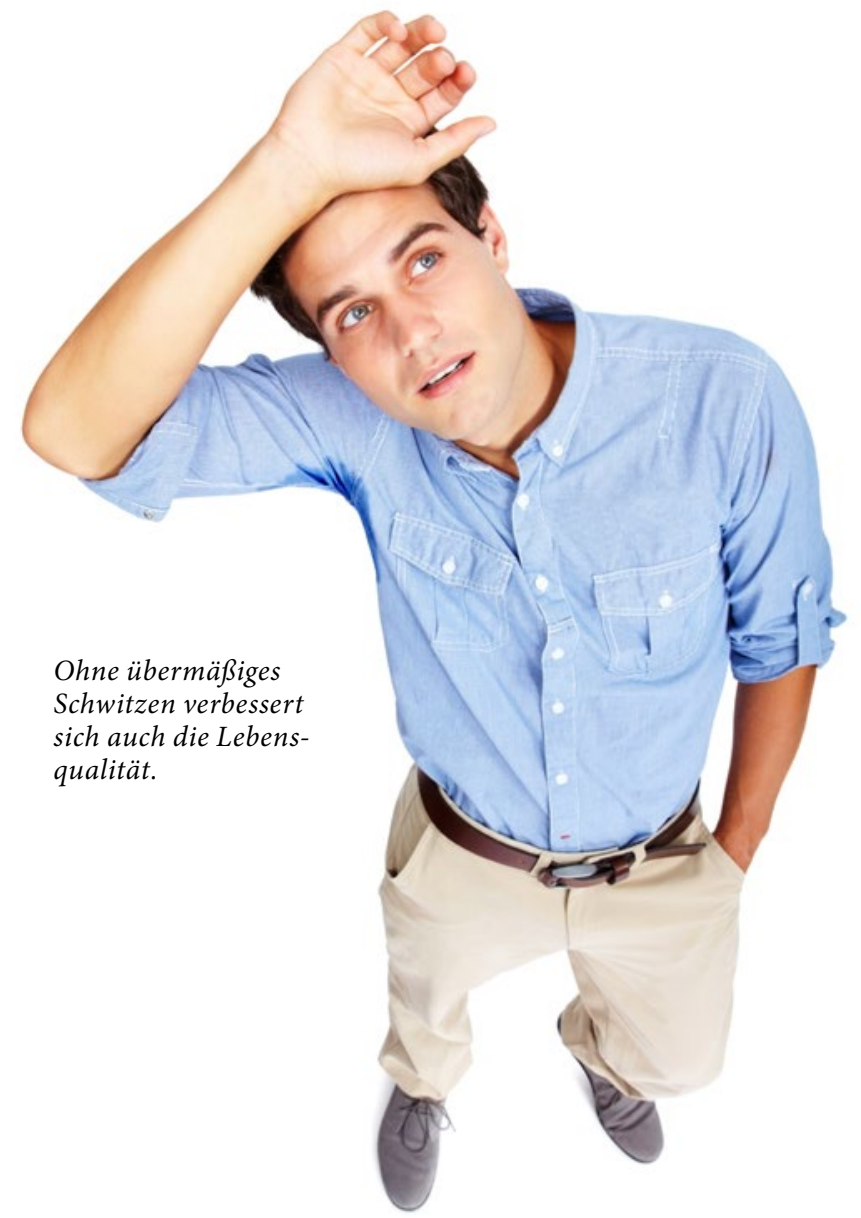

chen nach Therapiebeginn definiert. Nebenwirkungen und Lebensqualität (Dermatology Life Quality Index) wurden ebenfalls berücksichtigt.

\section{Verum verbessert Lebensqualität}

Nach 6 Wochen zeigte sich Oxybutynin dem Placebo überlegen ( $\mathrm{p}=0,009$ ): $67 \%$ der Patienten in der Verumgruppe, aber nur $27 \%$ in der Placebogruppe gaben an, nun weniger zu schwitzen als vor Studienbeginn. Von den 18 Verumpatienten, die eine Wirkung bestätigten, reduzierte sich der HDSS-Ausgangswert bei 5 Probanden um einen Punkt, bei 11 Probanden um 2 und bei 2 Probanden um 3 Punkte. Unter Placebo verbesserte sich der Wert bei 2 Probanden um 2 Punkte und bei 6 Probanden um einen Punkt. Auch schätzten die Patienten der Oxybutyningruppe ihre Lebensqualität nach 6 Wochen deutlich besser ein als die Patienten der Placebogruppe $(\mathrm{p}<0,01)$. Schwerwiegende Nebenwirkungen traten während der sechswöchigen Behandlung nicht auf. Fast die Hälfte der Patienten $(\mathrm{n}=13)$, die Oxybutynin eingenommen hatten, klagten aber über einen trockenen Mund, in der Placebogruppe waren es 3 Patienten.

\section{Erfolg unter Vorbehalt}

Oxybutynin könne, so die Schlussfolgerung der Studienautoren, die Symptome einer lokalisierten und generalisierten Hyperhidrose effektiv mildern und die Lebensqualität der Betroffenen verbessern, und das bei nur gering ausgeprägten Nebenwirkungen. Die kleine Patientenzahl, die kurze Beobachtungszeit und die Nicht-Verblindung schränken jedoch die Aussagekraft der Studie deutlich ein, wie die Studienautoren einräumen. Weder ließen sich anhand dieser Daten schwerwiegende Nebenwirkungen sicher ausschließen noch Aussagen über die langfristige Wirksamkeit und Verträglichkeit treffen.

Dr. Dagmar Kraus

Schollhammer M et al. Br J Dermatol 2015, Oct 14; DOI: 10.1111/bjd.13973

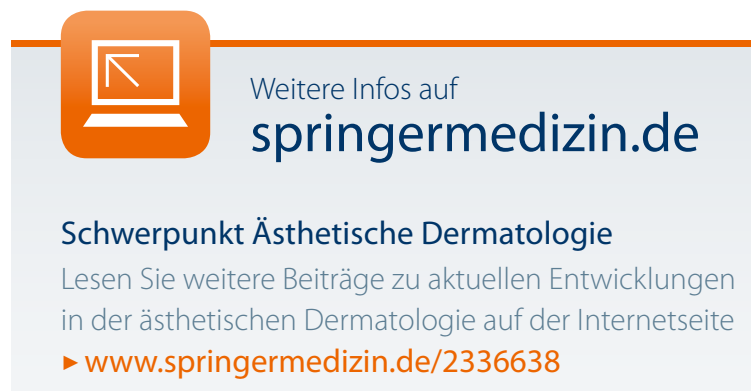

